\title{
Análise da ação de ácidos sobre ictiólitos da Formação Santana, Cretáceo Inferior da Bacia do Araripe, Nordeste do Brasil
}

\author{
Gabriel Teófilo Guedes Silva ${ }^{1}$ \\ Márcio Luiz da Silva ${ }^{2}$ \\ Eduardo Rodrigues de Oliveira ${ }^{3}$ \\ André Rodrigues Corrêa Gomes ${ }^{4}$ \\ IIFSULDEMINAS \\ Campus Inconfidentes \\ Praça Tiradentes, 416 Centro \\ Inconfidentes MG Brasil \\ CEP $37576-000$ \\ ${ }^{2}$ Departamento de Desenvolvimento \\ Educacional (DDE), \\ IFSULDEMINAS, \\ Campus Inconfidentes Praça Tiradentes, 416 \\ Centro \\ Inconfidentes MG Brasi \\ CEP $37576-000$ \\ ${ }^{3}$ Laboratório de Análise de Fertilidade de Solo \\ Departamento de Desenvolvimento Educacional \\ (DDE) \\ IFSULDEMINAS, \\ Campus Inconfidentes: \\ Praça Tiradentes, 416 Centro \\ Inconfidentes MG Brasil \\ CEP $37576-000$ \\ ${ }^{4}$ Laboratório de Química \\ Departamento de Desenvolvimento Educacional \\ (DDE) \\ IFSULDEMINAS \\ Campus Inconfidentes \\ Praça Tiradentes, 416 Centro \\ Inconfidentes MG Brasil \\ CEP $37576-000$ \\ *Autor correspondente \\ marcgeo10@yahoo.com.br
}

\begin{abstract}
RESUMO
Os fósseis são de grande importância para a construção do conhecimento da história da vida na Terra. Contudo, exigem muitos cuidados desde sua coleta em campo até a curadoria em laboratório e incorporação a uma coleção. A preparação, parte essencial desse processo, envolve a retirada dos fósseis da rocha matriz por meio de desgaste mecânico e/ou químico. Apesar da relevância de tal procedimento, o acesso ao seu conhecimento fica relativamente restrito aos laboratórios de paleontologia, havendo escassez desse tipo de publicação. Nesse sentido, o presente trabalho foi realizado com o objetivo de testar a utilização de diferentes tipos e concentrações de ácidos no processo de preparação de fósseis. Os testes com ácidos acético, clorídrico, fosfórico e sulfâmico foram executados em doze fragmentos de concreções calcárias (ictiólitos) da Formação Santana, Cretáceo Inferior da Bacia do Araripe. O ácido acético mostrou-se o mais adequado para o tratamento das concreções calcárias devido a sua ação prolongada e pouco agressiva.
\end{abstract}

Palavras-chave: ácido acético, ácido clorídrico, ácido fosfórico, ácido sulfâmico, curadoria paleontológica.

\section{ABSTRACT}

Fossils are of great importance for building knowledge of the history of life on Earth. However, it demands a careful handling from its collecting at the field to curatorship in laboratory and its incorporation in a collection. Preparation, a part of that process, evolves the removal of the fossils from the rock matrix, through mechanic and/or chemical attrition. Despite the relevance of such procedure, the access to its knowledge keeps itself relatively restricted to paleontology laboratories, lacking such type of publications. Then, this work was realized aiming to test the utilization of different types and concentrations of acids in preparation process of calcareous fossils. The tests with acetic, hydrochloric, phosphoric and sulfamic acids were executed in twelve fragments of calcareous concretions (ichthyolites) of the Santana Formation, Lower Cretaceous of the Araripe Basin. Acetic acid had showed itself the most appropriated for the treatment of calcareous concretions for its elongated and soft action.

Keywords: acetic acid, hydrochloric acid, phosphoric acid, sulfamic acid, paleontological curatorship.

\section{INTRODUÇÃO}

Os fósseis constituem tema de relevância, não só para questões de cunho paleontológico, mas para diversas outras áreas do conhecimento. Os estudos fossilíferos integram, também, um rol de disciplinas que possibilitam a introdução de temas de importância para o conhecimento das geociências e áreas afins (TEIXEIRA et al., 2006), além de fornecer dados e instrumentos para áreas diversas, tais como a educação e museologia, dentre outras.

Especificamente no tocante às coleções museológicas, os fósseis desempenham o papel ímpar de fazer mediação entre o público e um 
universo extinto pretérito, às vezes, desunido há milhões de anos. Dessa forma, os estudos paleontológicos podem apresentar atratividade para públicos diversos (MELLO et al., 2005, ALMEIDA et al., 2013; KELLNER, 2015). Contudo, a incorporação de um fóssil a uma coleção museológica requer um minucioso processo técnico e patrimonial conhecido como curadoria paleontológica (GREEN, 2001, SOCIEDADE DE HISTÓRIA NATURAL, 2019).

A curadoria paleontológica envolve uma série de cuidados desde a coleta dos fósseis até seu tratamento em laboratório e arquivamento em uma coleção científica ou exposição em espaço educacional, sendo um procedimento indispensável para assegurar a integridade física dos espécimes fósseis (CARVALHO, 2010; LEAL; BRITTO 2010; KELLNER, 2015; KEMNITZ; SANDFORD, 2015). Primeira das etapas da curadoria, a preparação consiste na retirada do sedimento sobrejacente ao espécime fóssil propriamente dito, de modo a expor ao máximo suas características anatômicas (KELLNER, 2015, SOCIEDADE DE HISTÓRIA NATURAL, 2019). Dentre as técnicas disponíveis para a preparação, pode-se adotar o método químico, que consiste no ataque à rocha matriz com ácidos, geralmente orgânicos (TOOMBS; RIXON, 1959). A preparação química é utilizada naquelas situações em que a abrasão mecânica da matriz não se faz pertinente. Isso se pode dar em função de o espécime possuir feições muito delicadas para choques físicos, por haver partes anatômicas inacessíveis a instrumentos manuais, dentre outros (GREEN, 2001).

O Instituto Federal de Educação, Ciência e Tecnologia do Sul de Minas Gerais (IFSULDEMINAS), Campus Inconfidentes participou de uma atividade didática de coleta de fósseis na Bacia do Araripe, Estado do Ceará, coordenada pela Universidade Federal do Rio de Janeiro (UFRJ). Cerca de 80 espécimes fósseis pertencentes a diversos grupos taxonômicos foram doados ao IFSULDEMINAS. Boa parte desse material corresponde a peixes em nódulos calcários (ictiólitos), reconhecidos por sua abundância nos afloramentos da Formação Santana, Cretáceo Inferior da Bacia do Araripe. A preparação desses fósseis exige o emprego de diversas técnicas de natureza química, devido ao material ser de difícil tratamento por abrasão mecânica, a exemplos dos realizados por Evander (1991, 2010).

Tendo em vista a existência de poucos trabalhos relacionados à preparação química de fósseis e à relevância do tema para a paleontologia, o presente trabalho teve como objetivo testar a utilização de diferentes tipos e concentrações de ácidos no processo de preparação de fósseis em concreções calcárias da Formação Santana, Cretáceo Inferior da Bacia do Araripe.

Espera-se que a presente pesquisa possa contribuir para a elucidação quanto ao emprego de diferentes ácidos no tratamento de fósseis em rochas calcárias similares às da Formação Santana.

\section{CARACTERIZAÇÃO DA ÁREA DE ESTUDOS}

A Bacia do Araripe (Figura 1) abrange parte do território do sul do Estado do Ceará e oeste do Estado do Pernambuco. Possui cerca de $12.200 \mathrm{Km}^{2} \mathrm{e}$ corresponde à maior área de rochas do Cretáceo expostas, dentre as bacias interiores do Nordeste do Brasil (CARVALHO; MELO, 2012). A origem dessas bacias está vinculada à abertura do Atlântico Sul e rift do Gondwana (Assine 2007, CARVALHO; MELO, 2012).

A estratigrafia da Bacia vem sendo intensamente discutida na literatura desde o início do Século XX. A esse respeito, Assine (1992) relaciona uma série de estudos com importantes atualizações na compreensão estratigráfica do Araripe até o final dos anos 1990. O autor (Op cit.) propõe a seguinte divisão: Formação Cariri (OrdovicianoSiluriano); Formações Brejo Santo e Missão
Velha (Jurássico); e formações Abaiara, Barbalha, Santana, Araripina e Exu (Cretáceo). Arai (2006) aponta para a problemática envolvida na concepção estratigráfica da Bacia do Araripe, atribuindo a Ponte e Appi (1990) e a Assine (1992), maior confiabilidade dentre os muitos autores divergentes. A partir desses trabalhos se têm importantes redefinições, como a organização da Bacia em dois grupos, a revisão da Formação Santana, dentre outras (PONTE; APPI 1990; ASSINE, 1992). As divergências entre esses autores são pequenas, mais associadas às questões terminológicas.

A Formação Santana, de onde foram extraídos os ictiólitos objeto dessa pesquisa, é composta essencialmente por calcários laminados, siltitos e argilitos (Membro Crato); gipsita e anidrita (Membro Ipubi); calcários, margas, siltitos e argilitos com nódulos carbo- 


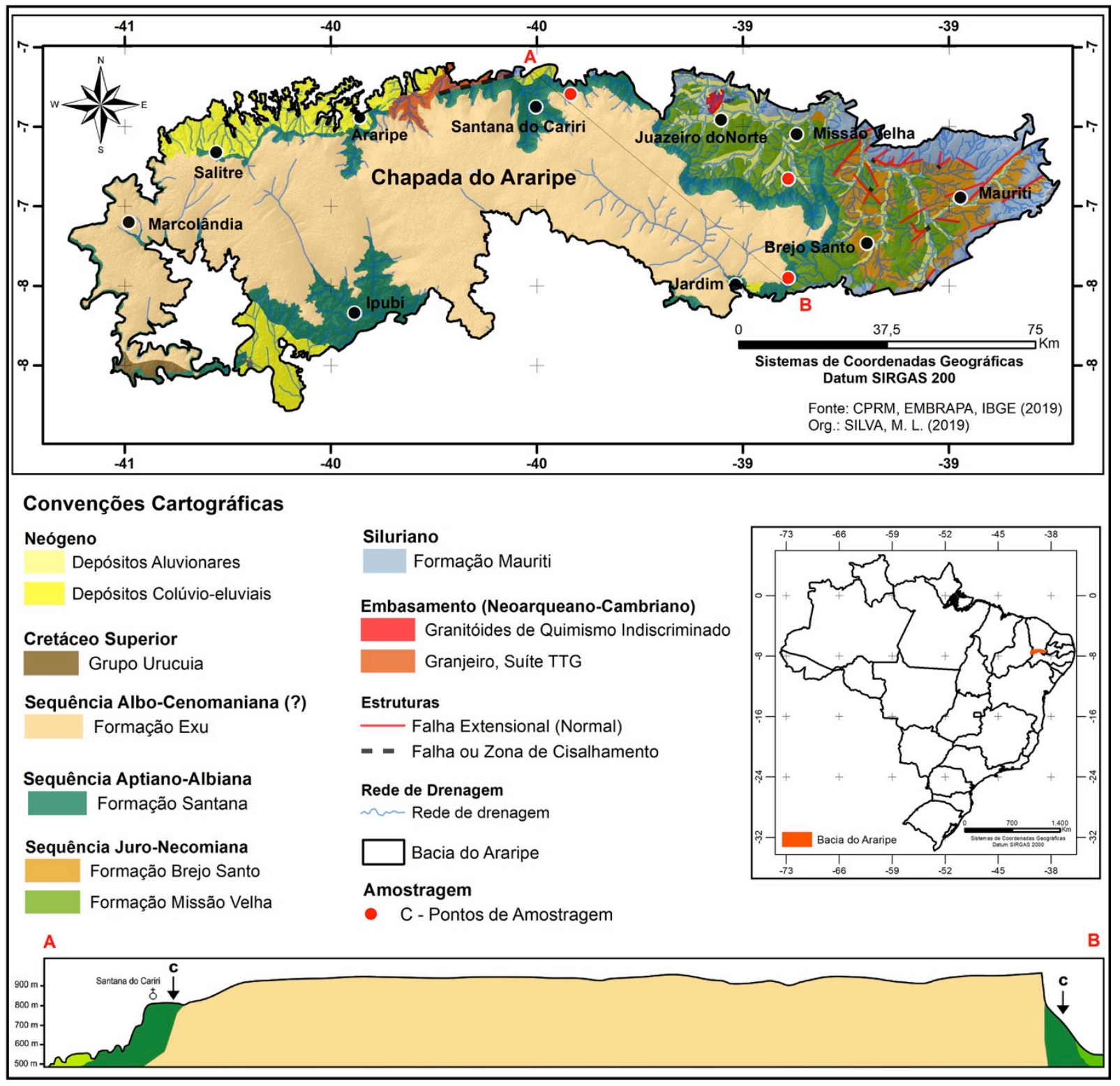

Figura 1

Mapa geológico simplificado da Bacia do Araripe.

náticos (Membro Romualdo) (ASSINE, 2007, CARVALHO; MELO, 2012). Possui idade compreendida entre o Neoaptiano e o Eoalbiano (Cretáceo Inferior) (Figura 1). Essa Formação constitui a de maior interesse paleontológico, em função da grande riqueza fossilífera (CARVALHO; SANTOS 2005; BOOS; VEJA, 2011).

Em se tratando do conteúdo fossilífero da

\section{MATERIAIS E MÉTODOS}

\subsection{AMOSTRAGEM}

Para a execução do presente trabalho, foi utilizada a estrutura física do Laboratório de Análises do Solo do IFSULDEMINAS, Campus Inconfidentes. $\mathrm{O}$ trabalho foi executado durante três dias consecutivos em setembro de 2018.
Bacia, há grande riqueza e diversidade de táxons. São encontrados fósseis de insetos, aracnídeos, moluscos, peixes, répteis atuais e extintos, vegetais, microfósseis (polens e esporos, dinoflagelados, foraminíferos, conchostráceos e ostracodes) e icnofósseis (ARAI; COIMBRA, 1990; CARVALHO; SANTOS, 2005; BRUNO; HESSEL, 2006; BOOS; VEJA, 2011; BARLING et al., 2014).
Foram preparados 12 béqueres com as soluções ácidas, e em cada recipiente se repousou uma amostra de rocha calcária (fragmentos de ictiólitos) com massa próxima a $3 \mathrm{~g}$ cada uma. 
As amostras foram extraídas de concreções calcárias hospedeiras de peixes fósseis (ictiólitos) da Formação Santana, Cretáceo Inferior da Bacia do Araripe. Essas rochas calcárias apresentam mineralogia compreendida por calcita e sílica, além de fosfatos e óxidos de ferro de forma subsidiária (MABESOONE, 1983).

\subsection{DETERMINAÇÕES ANALÍTICAS}

Os béqueres utilizados possuem $50 \mathrm{ml}$ de capacidade, dos quais 25 foram preenchidos com as soluções ácidas. As concentrações dessas foram de 5, 10 e 15\% para cada ácido empregado. A diluição foi executada em laboratório com o uso de capela, béqueres, pipetas e recipientes para armazenamento das soluções. Utilizaram-se os ácidos acético, clorídrico, fosfórico e sulfâmico. Esses ácidos são mencionados em diversos experimentos na literatura (BULLMAN, 1931; STEEN, 1931; TOOMBS; RIXON, 1959; GREEN，2001; CARVALHO 2010, PADILLA; PARRA, s.d., LEAL; BRITTO, 2010), exceto o ácido fosfórico, cuja adoção para a presente pesquisa se deu por sua disponibilidade no laboratório.

A massa das amostras foi medida periodicamente, a fim de verificar-se a efetividade da ação dos ácidos sobre a matriz por intervalo de tempo.

Para a análise da ação dos ácidos foram observadas intensidade da ação e sua uniformidade ao longo do tempo. A intensidade é medida pelo coeficiente de ação. Para tanto, tomou-se como modelo a ação do

\subsection{ANÁLISE ESTATÍSTICA}

Visando a avaliar o comportamento da ação dos ácidos sobre os ictiólitos em função do tempo, foi utilizado o cálculo de regressão geométrica. Considerando as especificidades desse experimento, a regressão geométrica foi selecionada para representar o consumo de rochas calcárias, a partir da aplicação de diferentes ácidos, no decorrer do tempo.

$\mathrm{O}$ modelo propõe que sejam $\mathrm{X}$ e $\mathrm{Y}$ duas variáveis, tal que $X$ possa ser utilizada para explicar Y. A relação é explicada por:

$$
Y_{i}=\beta_{0} X_{i}^{\beta i}+e_{i}
$$

onde $\beta_{0}$ e $\beta_{1}$ são os coeficientes do modelo estatístico, $i=1,2,3 \ldots$ é o índice que determina a ordem dos valores amostrais de X e Y. Já $\boldsymbol{e}_{i}$ é o erro associado ao modelo. Para estimar os
A extração dos fragmentos de rocha dos ictiólitos foi feita por meio de choque mecânico com martelo e ponteira, o que ocasionou amostragem pouco irregular (média de 3 g cada).

Optou-se por não utilizar os espécimes propriamente ditos para que não fossem danificados.

ácido acético, cuja utilização é amplamente aceita nos laboratórios de paleontologia. A uniformidade é dada pelo comportamento do ácido no decorrer do tempo de experimento, sendo demonstrada pela curva de regressão. Assim, aqueles ácidos cuja curva de regressão apresenta-se relativamente uniforme são considerados para o presente fim, eliminandose, porém, aqueles em que a curva de regressão mostra variações bruscas de valores de massa, ou consumo repentino de consideráveis quantias de massa. Tais fenômenos são interpretados como risco de destruição do espécime em preparação ou, em caso contrário, ação insuficientemente efetiva para o desgaste da matriz rochosa.

Após a verificação da ação ácida sobre as amostras, tabularam-se os valores para que se pudesse estabelecer a relação da efetividade do ácido sobre a rocha por intervalo de tempo. Durante a tabulação definiu-se a relação de consumo da rocha pelas soluções ácidas ao longo dos intervalos de tempo. A essa relação, chamou-se coeficiente de consumo, o qual é mensurado em gramas de rocha consumidos em intervalos de 1 hora.

valores de $\boldsymbol{\beta}_{0}$ e $\boldsymbol{\beta}_{1}$, deve-se linearizar a equação 1 , por meio da função $\ln$, chegando à seguinte equação:

$$
\ln Y_{i}=\ln \beta_{0}+\beta_{1} \ln X_{i}+e_{i}^{\prime}
$$

Segundo Gujarati (2000), uma forma de minimizar o erro associado ao modelo $\left(\boldsymbol{e}_{\boldsymbol{i}}{ }\right)$ é pelo método dos mínimos quadrados, em que se deve minimizar a seguinte expressão:

$$
\sum \widehat{e_{l}^{J^{2}}}=\sum\left(\ln Y_{i}-\widehat{\ln }_{l}\right)^{2}
$$

sendo que $\widehat{e_{l}}$ é o erro estimado do modelo (2), $\widehat{\operatorname{lnY}}_{l}$ é o valor estimado de $\ln Y_{i}$ pelo modelo de regressão. No caso do presente experimento, tem-se: 


$$
\widehat{\operatorname{lnY}}_{l}=\widehat{\ln \beta_{0}}+{\widehat{\beta_{1}}}_{1} \ln X_{i}
$$

onde $\widehat{\ln \beta_{0}}$ e $\widehat{\beta_{1}}$ são os valores estimados do modelo de regressão (2). A minimização do erro estimado do modelo (2) se dá pelas derivadas parciais da expressão (3) em função dos valores estimados dos modelos de regressão (2), igualadas a zero. Isso gerará um sistema de equações, que, solucionado, obterse-ão os valores estimados do modelo de regressão que minimizam o erro estimado do modelo (2).

De acordo com Gujarati (2000), o coeficiente de determinação $\left(\mathrm{R}^{2}\right)$ é uma medida que diz o quão bem a linha de regressão amostral se ajusta aos dados. Sua expressão é dada por:

$$
\frac{\sum\left(\widehat{Y}_{l}-\bar{Y}\right)^{2}}{\sum\left(Y_{i}-\bar{Y}\right)^{2}}
$$

onde $\bar{Y}$ é a média aritmética, dos valores amostrais de $\mathrm{Y}_{\mathrm{i}}$, e $\hat{\mathrm{Y}}_{\mathrm{i}}$ é o valor estimado de $\mathrm{Y}$, dado por:

$$
\widehat{Y_{l}}=\widehat{\beta_{0}} X_{i}^{\widehat{\beta_{1}}}
$$

$\mathrm{O}$ coeficiente de determinação $\left(\mathrm{R}^{2}\right)$ assumirá

\section{RESULTADOS E DISCUSSÃO}

Os resultados da ação dos ácidos acético, clorídrico, fosfórico e sulfâmico sobre os fragmentos de ictiólitos são apresentados abaixo (Tabela 1). Os intervalos de tempo foram irregulares, dadas as demais ações em execução no laboratório.

As curvas que apresentaram os melhores ajustes do coeficiente de regressão foram as do ácido sulfâmico a 10 e $15 \%$, e fosfórico a $5 \%$ (superiores a $90 \%$ ). O ácido acético a $15 \%$ teve o pior ajuste, dentre todos $(71,6 \%)$ (Tabela 2$)$. Isso significa que o modelo de regressão geométrica foi adequado para explicar o decréscimo da matriz rochosa em função do tempo, já que o coeficiente de determinação foi superior a $80 \%$ na maioria dos casos. Os valores estimados de $\beta_{0}$ e $\beta_{1}$ foram significativos a $5 \%$ em quase todos os experimentos, com exceção para o $\beta_{0}$ estimado para a curva de ácido sulfâmico a $15 \%$, cujo valores reais entre 0 e 1 . Quanto mais próximo de 1, melhor é o ajuste da regressão.

Nesse modelo, necessário se faz testar a hipótese de significância dos coeficientes do modelo. O erro tipo I $(\alpha)$ é a probabilidade de rejeitar a hipótese nula, quando esta é verdadeira. Pode-se apresentar a probabilidade de significância, ou nível descritivo, ou ainda p-valor do teste (BUSSAB; MORETTIN, 2010). Utilizou-se o teste t de Student. O citado modelo permite a compreensão do decréscimo da massa de rocha calcária em função do tempo. Como cada experimento se inicia no instante zero, gerar-se-ia um erro ao tentar ajustar os dados ao modelo selecionado. Assim, para se determinarem os coeficientes de regressão, os coeficientes de determinação e as curvas de regressão, houve uma correção dos valores amostrais da variável tempo, sendo:

$$
\text { Tempo Corrigido }=\text { Tempo }+0,01
$$

Além disso, para cada concentração ácida foi estimada a curva de regressão e seus respectivos coeficientes de regressão e determinação. Para as análises foram utilizados o Calc do Libre Office (versão 5.3.0.3) e o software $R$ (versão 3.5.3).

p-valor foi superior a $5 \%$.

Os ácidos apresentaram graus de efetividade sobre a matriz rochosa. A efetividade de cada ácido nas diferentes concentrações foi relevante, por indicar a eficiência na preparação dos fragmentos de calcário e, por apresentar baixo risco para o espécime propriamente dito, quando houver necessidade real da preparação química de um fóssil.

O ácido acético apresentou comportamento consideravelmente constante, demonstrando pequenas variações entre as diferentes concentrações e intervalos de tempo. $\mathrm{O}$ coeficiente de consumo apresentou baixa variação (Tabela 3). As curvas de regressão apresentaram comportamento de variação suave no decorrer do tempo de experimento, evidenciando sua previsibilidade de ação (Figura 2). 
Tabela 1 - Ação das soluções ácidas sobre as amostras de rochas calcárias (fragmentos de ictiólitos)

\begin{tabular}{|c|c|c|c|c|c|c|c|c|c|c|c|c|c|c|c|}
\hline \multirow{2}{*}{$\mathbf{N}^{\mathbf{o}}$} & \multirow{2}{*}{ Data } & \multirow{2}{*}{ Hora } & \multirow{2}{*}{$\begin{array}{c}\text { Tempo } \\
\text { parcial } \\
\text { (min.) }\end{array}$} & \multirow{2}{*}{$\begin{array}{c}\text { Tempo } \\
\text { total } \\
\text { (min.) }\end{array}$} & \multicolumn{11}{|c|}{ Massa da amostra (g) } \\
\hline & & & & & A5 & A10 & A15 & C5 & $\mathrm{C} 10$ & C15 & F5 & F10 & F15 & S5 & S10 \\
\hline 1 & 21 & $9 \mathrm{~h} 32$ & 0 & 0 & 3,15 & 3,07 & 3,18 & 3,37 & 2,91 & 3,64 & 2,96 & 3,01 & 3,88 & 2,73 & 3,33 \\
\hline 2 & 21 & $9 \mathrm{~h} 42$ & 10 & 10 & 3,13 & 3,02 & 3,12 & 3,07 & 2,17 & 2,3 & 2,66 & 2,46 & 3,02 & 2,72 & 2,52 \\
\hline 3 & 21 & $9 \mathrm{~h} 57$ & 15 & 25 & 3,08 & 2,98 & 3,06 & 2,98 & 1,95 & 2,12 & 2,56 & 2,25 & 2,69 & 2,67 & 2,26 \\
\hline 4 & 21 & $10 \mathrm{~h} 4$ & 47 & 72 & 2,92 & 2,77 & 2,8 & 2,87 & 1,67 & 1,94 & 2,32 & 1,74 & 1,91 & 2,58 & 1,75 \\
\hline 5 & 21 & $11 \mathrm{~h} 0$ & 25 & 97 & 2,85 & 2,7 & 2,7 & 2,85 & 1,65 & 1,93 & 2,28 & 1,68 & 1,84 & 2,57 & 1,67 \\
\hline 6 & 21 & $12 \mathrm{~h} 0$ & 53 & 150 & 2,75 & 2,57 & 2,53 & 2,85 & 1,64 & 1,92 & 2,27 & 1,62 & 1,76 & 2,56 & 1,59 \\
\hline 7 & 21 & $13 \overline{\mathrm{h}} 4$ & 95 & 245 & 2,67 & 2,4 & 2,32 & 2,85 & 1,64 & 1,92 & 2,26 & 1,61 & 1,74 & 2,57 & 1,52 \\
\hline 8 & 21 & $15 \mathrm{~h}$ & 80 & 325 & 2,59 & 2,33 & 2,17 & 2,84 & 1,64 & 1,9 & 2,25 & 1,59 & 1,73 & 2,54 & 1,51 \\
\hline 9 & 21 & $16 \mathrm{~h} 0$ & 65 & 390 & 2,53 & 2,26 & 2,07 & 2,83 & 1,63 & 1,89 & 2,24 & 1,58 & 1,71 & 2,54 & 1,5 \\
\hline 10 & 21 & $18 \mathrm{~h}$ & 115 & 505 & 2,49 & 2,18 & 1,97 & 2,85 & 1,62 & 1,9 & 2,23 & 1,57 & 1,72 & 2,54 & 1,5 \\
\hline 11 & 22 & 16h1 & 0 & 505 & 2,32 & 1,95 & 1,37 & 2,83 & 1,61 & 1,9 & 2,23 & 1,57 & 1,67 & 2,53 & 1,45 \\
\hline 12 & 22 & $16 \mathrm{~h} 3$ & 25 & 530 & 2,26 & 1,85 & 1,27 & 2,43 & 0,95 & 0,83 & 1,89 & 1,37 & 1,38 & 2,44 & 0,95 \\
\hline 13 & 22 & $1 \overline{7 h}$ & 25 & 555 & 2,19 & 1,78 & 1,18 & 2,25 & 0,66 & 0,44 & 1,5 & 1,09 & 0,82 & 2,36 & 0,67 \\
\hline 14 & 22 & 17h3 & 33 & 588 & 2,14 & 1,72 & 1,12 & 2,18 & 0,46 & 0,23 & 1,22 & 0,81 & 0,37 & 2,32 & 0,47 \\
\hline 15 & 22 & $21 \mathrm{~h} 2$ & 232 & 820 & 1,82 & 1,3 & 0,67 & 2,11 & 0,2 & 0,01 & 0,71 & 0,15 & 0 & 2,27 & 0,03 \\
\hline 16 & 25 & $1 \overline{1 h}$ & 58028 & 5848 & 1,47 & 0,6 & 0 & 2,11 & 0,17 & 0 & 0,67 & 0 & 0 & 2,26 & 0 \\
\hline
\end{tabular}

Os intervalos correspondem a períodos de tempo entre pesagens da massa das amostras de rocha. O tempo total corresponde ao período de tempo em que as rochas estiveram sujeitas à ação dos ácidos. Esse período somente foi interrompido para que se pesassem as amostras e nos intervalos de um dia para outro. As massas das amostras não apresentam regularidade, devido à dificuldade de fragmentação precisa do material. O nome dado às amostras compõe-se da letra inicial do ácido e sua concentração (e.g. A5 = ácido Acético a [5\%]; F10 = ácido fosfórico a [10\%]).

Tabela 2 - Valores dos coeficientes estimados de regressão e dos coeficientes de determinação para as curvas de regressão da massa em função do tempo corrigido para os ácidos acético, clorídrico, fosfórico e sulfâmico

\begin{tabular}{|c|c|c|c|c|c|}
\hline Ácido & $\beta_{0}$ estimado & p-valor $\left(\ln \left(\beta_{0}\right)\right.$ estimado $)$ & $\beta_{1}$ estimado & p-valor $\left(\beta_{1}\right.$ estimado $)$ & $R^{2}(\%)$ \\
\hline Acético $(5 \%)$ & 2,81074052 & $7,53 \mathrm{E}-14$ & $-0,041738651$ & 0,000121 & 82,12944 \\
\hline Acético $(10 \%)$ & 2,611691037 & $6,76 \mathrm{E}-12$ & $-0,063788539$ & 0,000186 & 80,357845 \\
\hline Acético $(15 \%)$ & 2,5236973 & $5,40 \mathrm{E}-09$ & $-0,096617167$ & 0,00103 & 71,55851 \\
\hline Clorídrico $(5 \%)$ & 2,9527419 & $<2,00 \mathrm{E}-016$ & $-0,2277139$ & $2,53 \mathrm{E}-05$ & 87,30239 \\
\hline Clorídrico $(10 \%)$ & 1,863827486 & $3,12 \mathrm{E}-10$ & $-0,07882928$ & $2,31 \mathrm{E}-05$ & 87,55414 \\
\hline Clorídrico $(15 \%)$ & 2,15751979 & $6,06 \mathrm{E}-10$ & $-0,07921621$ & $2,00 \mathrm{E}-04$ & 80,05623 \\
\hline Fosfórico (5\%) & 2,421467 & $7,73 \mathrm{E}-15$ & -0.04054375 & $5,95 \mathrm{E}-06$ & 90,76288 \\
\hline Fosfórico $(10 \%)$ & 1,9157264 & $4,77 \mathrm{E}-10$ & $-0,096881$ & $9,09 \mathrm{E}-06$ & 89,86228 \\
\hline Fosfórico $(15 \%)$ & 2,8249769 & $8,83 \mathrm{E}-10$ & $-0,12400237$ & $9,48 \mathrm{E}-06$ & 89,766811 \\
\hline Sulfâmico (5\%) & 2,6085952 & $<2,00 \mathrm{E}-16$ & $-0,0119448$ & $3,50 \mathrm{E}-05$ & 86,37069 \\
\hline Sulfâmico (10\%) & 1,90463515 & $3,47 \mathrm{E}-10$ & $-0,1192736$ & $1,11 \mathrm{E}-06$ & 93,62863 \\
\hline Sulfâmico $(15 \%)$ & 0,9923058 & 0,83 & $-0,177136904$ & $1,74 \mathrm{E}-06$ & 92,959674 \\
\hline
\end{tabular}

Tabela 3. Coeficiente de consumo dos ácidos nas diferentes concentrações testadas

\begin{tabular}{cccc}
\hline Ácido & \multicolumn{3}{c}{ Coeficiente de consumo para cada concentração (g/min). } \\
\cline { 2 - 4 } & $\mathbf{5 \%}$ & $\mathbf{1 0 \%}$ & $\mathbf{1 5 \%}$ \\
\hline Acético & 1,02 & 1,5 & 1,98 \\
Clorídrico & 0,78 & 1,68 & 2,22 \\
Fosfórico & 1,38 & 1,86 & 5,04 \\
Sulfâmico & 0,3 & 2,04 & 4,14 \\
\hline
\end{tabular}

O ácido clorídrico demonstrou mudanças significativas de comportamento entre as distintas concentrações, variando acentuadamente os valores do coeficiente de ação. Os resultados se distanciaram dos do ácido acético. $\mathrm{O}$ ácido clorídrico mostrou-se inicialmente menos efetivo que o anterior, aumentando-se, porém, sua intensidade de ação rapidamente conforme se aumentou a concentração da solução (Tabela 3 ). As curvas de regressão confirmaram a baixa previsibilidade do ácido, exibindo mudanças abruptas de valor, ora no tempo, ora na massa
(Figura 2). Dessa forma, o ácido clorídrico foi insuficientemente efetivo sobre a matriz calcária, a $5 \%$ e agressivo nas demais concentrações.

O ácido fosfórico, assim como o clorídrico, apresentou variações indesejáveis para 0 proposto fim. Os coeficientes de consumo alcançaram os valores mais elevados dentre todos (Tabela 3). As curvas de regressão apresentaram variações geométricas excessivas em se tratando da preparação de fósseis (Figura 2). 


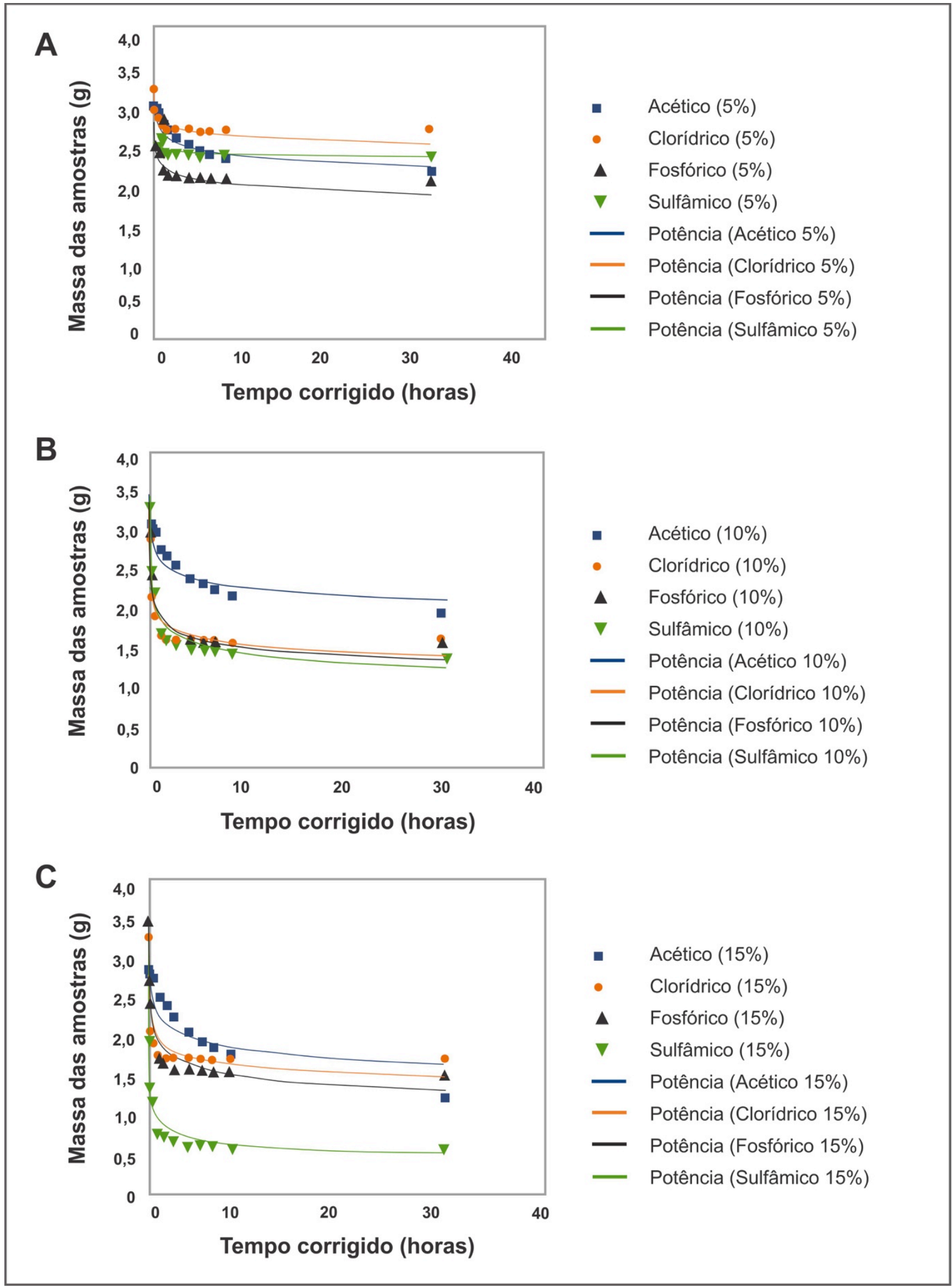

Curvas de regressão da massa (em gramas) em função do tempo corrigido (em horas) dos ácidos acético, clorídrico, fosfórico e sulfâmico. A) Concentração de 5\%; B) Concentração de 10\%; C) Concentração de $15 \%$.

Por fim, para o ácido sulfâmico os coeficientes de consumo também tiveram variações muito elevadas (Tabela 3 ). As curvas de regressão apresentaram comportamentos instáveis para o ataque químico aos fósseis de rochas calcários (Figura 2). Na concentração 
de 5\% não houve, praticamente, consumo da rocha, enquanto que nas demais se chegaram a superar o ácido clorídrico e a quase igualar o fosfórico, de maior coeficiente.

Nesse sentido, o ácido acético teve o comportamento mais apropriado para a preparação dos ictiólitos. A previsibilidade de seu comportamento entre as diferentes concentrações permitiu que se alterasse a concentração conforme a necessidade da condição apresentada pelo espécime, o que pôde otimizar o tempo de trabalho no laboratório, corroborando a pertinência de seu uso, já reconhecida pela literatura (TOOMBS; RIXON, 1948, 1959; CARVALHO, 2010, LEAL; BRITTO, 2010).

Os ácidos clorídrico e fosfórico não demonstraram potencial de uso para a preparação dos ictiólitos. Ambos apresentaram mudanças de comportamento acentuadas diante das variações experimentais, diferentemente do ácido acético. Essas variações se deram, ora incluindo o risco de dano físico ao espécime, ora não havendo efetividade no desgaste da matriz rochosa. Pelos resultados apresentados e interpretados, esses ácidos poderiam ter aplicação em casos cuja matriz calcária representasse uma parcela muito grande do espécime, não havendo assim, risco desse ser comprometido pela ação ácida.

Igualmente, o ácido sulfâmico não apresentou ação eficiente ou adequada para a rocha calcária, o que contraria os resultados obtidos por Padilla e Parra (s.d.). Isso pode ser devido a uma complexa combinação de variáveis como a composição da matriz, processo de fossilização, propriedades do fóssil, ambiente de fossilização, dentre outros (CARVALHO, 2010). Haja vista os resultados positivos encontrados pelos autores, compreende-se que mais estudos utilizando o ácido sulfâmico devem ser realizados.

Carvalho (2010) recomenda o uso de ácido acético, salientando sua elevada eficiência no

\section{CONCLUSÃO}

O ácido acético mostrou-se o mais indicado para a preparação química de concreções calcárias da Formação Santana. Os demais ácidos testados, ou seja, os ácidos clorídrico, fosfórico e sulfâmico, não apresentaram comportamento adequado para a matriz rocho- processo de preparação de fósseis. Há, contudo, registros de resultados ainda mais eficientes utilizando o ácido fórmico (RIXON, 1949; EVANDER, 1991; LEAL; BRITTO, 2010). Esse ácido apresenta ação mais efetiva sobre a rocha calcária sem, contudo, atacar o fosfato de cálcio presente nas estruturas ósseas do espécime. $\mathrm{O}$ contraponto seria o risco oferecido à saúde quando do uso desse último, o que não ocorreria com a adoção do ácido acético (LEAL; BRITTO, 2010). Toombs e Rixon (1948, 1959), pioneiros das atuais técnicas de preparação química de fósseis, por outro lado, afirmam o desconhecimento de casos em que o ácido acético teria sido agressivo ao espécime fóssil, mesmo após dezenas de anos de testes com a preparação química dos diversos táxons de vertebrados de diferentes períodos geológicos.

Alerta-se, entretanto, para a essencialidade de um produto que proteja o espécime (TOOMBS; RIXON, 1948, 1959; GREEN, 2001; LEAL; BRITTO, 2010; NIZER; WEINSCHÜTZ, 2014; PADILLA; PARRA, s.d.), especificamente, nesse caso, polibutil metacrilato, popularmente conhecido como paraloide.

A partir dos dados literários consultados e dos testes realizados sobre rochas calcárias e suas interpretações, enfatiza-se, neste trabalho, que o ácido acético foi o mais apropriado dentre aqueles testados para a preparação de ictiólitos da Formação Santana. Ressalta-se, não obstante, que a preparação de fósseis por meio do ataque químico às rochas também pode ser realizado com o uso de outros compostos e metodologias, o que requer, contudo, que mais ações sejam publicadas na literatura para que se compreenda melhor o comportamento dos materiais utilizados (BULLMAN, 1931; STEEN, 1931; TRIPLEHORN et al., 2002; VASCONCELOS et al., 2016).

sa utilizada.

Recomenda-se, dessa forma, a utilização do ácido acético no tratamento químico de ictiólitos com características similares aos espécimes da Formação Santana, Cretáceo Inferior da Bacia do Araripe. 


\section{AGRADECIMENTOS}

Os autores agradecem ao Núcleo de Incentivo à Pesquisa e Extensão (NIPE) do IFSULDEMINAS Campus Inconfidentes, pelo apoio financeiro; ao Laboratório de Análise de Fertilidade de Solo (Campus Inconfidentes) pela cessão do espaço físico e apoio nas determinações analíticas; ao Prof. Dr. Ismar Carvalho (UFRJ) por autorizar a participação e ceder os espécimes ao IFSULDEMINAS; à Dra. Aline Ghilardi, pela colaboração no

\section{REFERÊNCIAS}

ALMEIDA L.F; ZUCON M. H.; SOUZA J.F.; REIS V.S.; VIEIRA F.S. 2013. Ensino de Paleontologia: uma abordagem não-formal no Laboratório de Paleontologia da Universidade Federal de Sergipe. Terrae Didatica. 10:14-21.

ARAI M. 2006. Revisão estratigráfica do Cretáceo Inferior das bacias interiores do Nordeste do Brasil. Geociências. 25(1):7-15.

ARAI M.; COIMBRA J.C. 1990. Análise paleoecológica do registro das primeiras ingressões marinhas na Formação Santana (Cretáceo Inferior da Chapada do Araripe). Atas do I Simpósio sobre a Bacia do Araripe e Bacias Interiores do Nordeste, Crato.

ASSINE M.L. 1992. Análise estratigráfica da Bacia do Araripe, Nordeste do Brasil. Rev.Bras. Geoc.. 22(3):289-300.

ASSINE M.L. 2007. Bacia do Araripe. B. Geoci. Petrobras. 15(2):371-389.

BOOS A.D.S.; VEGA C.S. 2011. Paleofauna de vertebrados registrada na Formação Santana (Cretáceo), Bacia do Araripe, nordeste do Brasil. Acta. Biol. Par. 40(3-4):119-127.

BRUNO A.P.S.; HESSEL M.H. 2006. Registros paleontológicos do Cretáceo marinho da Bacia do Araripe. Estudos Geológicos. 16(1):30-49.

BULLMAN O.M.B. 1931. Note on Palaeospondylus gunni Traquair. Ann. Mag. Nat. Hist. 10:179-190.

BUSSAB, W.O; MORETTIN, P.A. 2010. Estatística Básica. São Paulo, Editora Saraiva. 568 pp.

CARVALHO I.S. 2010. Curadoria Paleontológica. In: I. Carvalho de S. (ed). Paleontologia: conceitos e métodos. Rio de Janeiro, Editora Interciência. p.: 373-386.

CARVALHO I.S.; MELO J.H.G. 2012. Bacias interiores do Nordeste. In: Hasui Y.; Carneiro C.D.R.; Almeida F.F.M. \& Bartorelli A. (org.). Geologia do Brasil. São Paulo, Editora Beca, p.:502-509.

CARVALHO M.S.S.; SANTOS M.E.C.M. 2005. Histórico das pesquisas paleontológicas na bacia do Araripe, Nordeste do Brasil. Anuário do Instituto de Geociências. 28(1):15-34

DE CARVALHO, G.K.R.; HESSEL, M.H.; ARAÚJO, A.L. 2012. Himenópteros e a Bacia projeto de pesquisa do qual a presente ação é resultante; ao Prof. Dr. Marcos Magalhães, Coordenador do Laboratório de Zoologia, pelo apoio no desenvolvimento da escrita do trabalho; ao Prof. Dr. Jorge dos Santos, do Laboratório de Química, pelas indicações quanto ao experimento com os ácidos; à estudante de graduação em Ciências Biológicas, Syara de Noronha, pelo apoio na execução do experimento.

do Araripe. Revista de Geologia. 24(2):150-171.

EVANDER R.L. 1991. Standard preparation technique for fossil fish from the Romualdo Member of the Santana Formation. Department of Vertebrate Paleontology, American Museum of Natural History, New York, 10pp.

EVANDER R.L. 2010. Special methods of acid preparation. Department of Vertebrate Paleontology, American Museum of Natural History, New York, 5pp.

GREEN O.R. 2001. A manual of practical laboratory and field techniques in Palaeobiology. Department of Earth Sciences, University of Oxford, United Kingdom, 546 pp.

GUJARATI, D. 2006. Econometria Básica. Rio de Janeiro, Editora Saraiva, 400pp.

JUNIOR, F.C.B.; MARQUES, R.B. 1997. Um novo lagarto do Cretáceo do Brasil (Lepidosauria, Squamata, Lacertilia - Formação Santana, Aptiano da Bacia do Araripe). Anuário do Instituto de Geociências. 20:233-240.

KELLNER A.W.A. 2015. Do campo ao laboratório: a viagem de um fóssil. In: M.B. Soares (org.). A Paleontologia na Sala de Aula. Ribeirão Preto. Sociedade Brasileira de Paleontologia, p.: 398-418.

KEMNITZ C.; SANDFORD S. 2015. Care of fossil, mineral and rock collections. Linking natural science collections in Wales, $3 \mathrm{pp}$.

LEAL, M.E.C.; BRITO, P.M. 2004. Técnicas de preparação química para vertebrados fósseis, In: CARVALHO, I.S. (ed.) Paleontologia. Editora Interciência, p.43-50.

MABESOONE, J.M. 1983. Sedimentologia, Recife, Editora Universitária (UFPE), 475p.

MELLO F.T.; MELLO L.H.C. \& TORELLO M.B.F. 2005. A Paleontologia na educação infantil: alfabetizando e construindo o conhecimento. Ciência \& Educação. 11(3):395410

NIZER M.W.; WEINSCHÜTZ L.C. 2014. Coleta e preparação de exemplares fósseis de Caiuajara dobruskii do Cretáceo da Bacia Sedimentar do Paraná. Ci. Biol. Saúde. 20(2):131-134.

PADILLA C. B.; PARRA M.L. s.d. Acid preparation of fossils using sulfamic acid, a weak organic acid, and its advantages over 
acetic and formic acid preparation. Fundación Colombiana de Geobiología. Documento não publicado.

PONTE F.C.; APPI C.J. 1990. Proposta de revisão da coluna litoestratigráfica da Bacia do Araripe. In: CONGRESSO BRASILEIRO DE GEOLOGIA. Sociedade Brasileira de Geologia. 1:211-226.

$\mathrm{R}$ CORE TEAM 2018. R: A language and environment for statistical computing. R Foundation for Statistical Computing, Vienna, Austria.

RIXON A.E. 1949. The use of acetic and formic acids in the preparation of fossils. Mus. Jour. 49:116.

SOCIEDADE DE HISTÓRIA NATURAL. 2019. Preparação de fósseis. Disponível em: http://www.shn.pt/preparacao-de-fosseis. Acessado em 27 fev 2019.

STEEN, M.C. 1931. The British Museum collection of Amphibia from the middle coal measures of Linton, Ohio. Proc. Zool. Soc. London. p.849-891.

TEIXEIRA W.; FAIRCHILD T.R.; TOLEDO M.C.M. \& TAIOLI F. 2009. Decifrando a Terra. São Paulo, Editora Nacional. 623 pp.

TOOMBS H. A.; RIXON A. E. 1948. The use of acetic acid in the development of vertebrate fossils. Mus. Jour. 48:54-55.

TRIPLEHORN D.M.; BOHOR B.F.; BETTERTON W.J. 2002. Chemical disagreggation of kaolinitic claystones (tonsteins and flint clays). Clays and Clay Minerals. 50(6):766-770.

TOOMBS, H. A.; RIXON, A. E. 1959. The use of acids in the preparation of vertebrate fossils. Department of Paleontology, British Museum (Natural History). 9 pp.

VASCONCELOS A.G.; SANTOS L.V.; KRAEMER B.M. 2016. Preparação físicoquímica de esqueleto submerso encontrado na Caverna Poço Azul, Bahia. Terrae Didatica. 12(3):163-171. 